\title{
Transmission Loss Reduction of Circular Terahertz Waveguide Using Dielectric-Lined Method
}

\author{
Achmad Munir and Aryan Setiawan \\ Radio Telecommunication and Microwave Laboratory \\ School of Electrical Engineering and Informatics \\ Institut Teknologi Bandung, Jalan Ganesha 10 Bandung 40132, Indonesia \\ munir@ieee.org
}

\begin{abstract}
In this paper, the transmission loss reduction for circular terahertz waveguide is investigated using dielectric-lined method. As is well known, the guided terahertz radiation suffers from high transmission loss due to the increase of ohmic loss and the lack of dielectric materials which has sufficiently transparency. As the existence of transmission loss, the quality of communication is therefore affected thoroughly. Here, the modification of hollow circular metallic terahertz waveguide by coating the inner surface of waveguide walls using some dielectric material, i.e. polystyrene, called as dielectric-lined method is proposed to reduce the transmission loss. The investigation is performed at $\mathrm{THz}$ frequencies from $0.1 \mathrm{THz}$ to $1.0 \mathrm{THz}$ including the analysis of transmission loss for each resonance mode and frequency band. The result shows that the proposed method with $15 \mu \mathrm{m}$ thickness of polystyrene coating is successfully decreasing the transmission loss for the $\mathrm{TE}_{01}$ wave mode below $1 \mathrm{~dB} / \mathrm{m}$ with smaller amplitude of electric field around the inner surface of waveguide walls. Furthermore, the discussion of investigation results for diameter variation and different thicknesses of polystyrene coating is also presented.
\end{abstract}

Keywords: circular terahertz waveguide, dielectric-lined, resonance mode, transmission loss reduction

\section{Introduction}

In recent times, the electromagnetic waves radiation in terahertz $(\mathrm{THz})$ as well as in optical frequencies has been implemented for wide range applications [1]-[7]. In spectroscopy domain, this radiation could enhance the sensitivity of spectroscopy with resolution less than $1 \mathrm{~mm}$ [1]. It is also applicable for 3D imaging application of structures and materials [2]-[3]. In addition, terahertz radiation has relatively low photon energy for damaging tissues and DNA so that it is widely used in medical imaging [4]. Some example of its application is in medical for 3D imaging phantom [5]. While in [6]-[7], terahertz 3D imaging system has been investigated for interferometry and FMCW millimeter wave radar.

As is well-known, in $\mathrm{THz}$ frequency which ranges from microwaves to visible light frequencies, there are no licenses which have been distributed up to now. This means that it is an empty band which is usable for wireless communications [8]. Actually, this band as well as the optical wave band has a few good properties, such as high transmission rate, well directional, high security, small scattering and good penetrating signal. Moreover, the band can also provide high quality of mobile broadband communication for multimedia services at $10 \mathrm{Gbps}$. In early of its development, the analysis of terahertz wave propagation has adapted the solution offered by the guided wave for radio and optic. Both solutions for coaxial metallic waveguide and dielectric circular fiber suffered in high transmission loss [9]-[10]. The reasons of these poor performances are caused by the increase of ohmic loss in metals at $\mathrm{THz}$ frequencies and lack of good transparency of dielectric materials. Therefore a lot of researches have been conducted to find better solutions for $\mathrm{THz}$ transmission. One of solutions to obtain better transmission loss is by using a dielectric-lined hollow circular metallic waveguide [11][13].

Received: June $3^{\text {rd }}, 2013$. Accepted: September $6^{\text {th }}, 2013$ 
Inspiring from the loss reduction using dielectric-lined method, in this paper, the solution is therefore proposed to be applied for $\mathrm{THz}$ waveguide with the focus investigation performed at frequency of $0.1 \mathrm{THz}$ up to $1.0 \mathrm{THz}$. By coating the inner surface of hollow circular metallic terahertz waveguide wall using some dielectric material, the change in transmission parameters is observed and analyzed. All of transmission parameters will be obtained and calculated using Finite Element Method (FEM). The paper is organized as follows: at first the basic theory related to hollow circular waveguide will be described briefly. The resonance modes of waveguide and its transmission loss are also included in the description. Then, the investigation of transmission loss is carried out and followed by introducing the dielectric-lined method for loss reduction. Some results for different diameter and variation thickness of dielectric material coating are analyzed to determine the optimum transmission loss. The discussion related to the investigation results will be presented consecutively and then followed by the conclusion.

\section{Circular Waveguide}

\section{A. Cut-off Frequency and Resonance Mode}

A hollow metallic circular terahertz waveguide utilizes conducting metal to be able to transform light wave by reflections from the walls. The electric and magnetic fields associated with electromagnetic waves that propagate through the medium characterized by permeability $(\mu)$ and permittivity $(\varepsilon)$ satisfy source-free Maxwell's equations. A circular waveguide has particular field patterns that travel in the direction of propagation referred as wave mode. It is a solution of wave equation solved with specific boundary conditions. A waveguide itself supports the Transverse Electric (TE) wave mode with no electric field component in the propagation direction $\left(E_{\mathrm{z}}=0, H_{\mathrm{z}} \neq 0\right)$, and the Transverse Magnetic (TM) wave mode with no magnetic field component in the propagation direction $\left(E_{\mathrm{z}} \neq 0, H_{\mathrm{z}}=0\right)$. It notes that the electric and magnetic fields in this case are assumed propagating in $z$ direction.

An ideal waveguide which travels the signal without loss has only imaginary part of propagation constant $(\alpha=0, \gamma=j \beta)$, otherwise propagation constant is complex-valued. In a waveguide, the wave mode propagates only if the propagation constant has imaginary value and the source of signal operates at a frequency higher than its cut-off frequency. The cut-off frequency of TE and TM wave modes in a circular waveguide of radius $a$ and filled by some dielectric $\left(\varepsilon_{r}, \mu_{r}\right)$ are given by (1) and (2), respectively [14].

$$
\begin{aligned}
& f_{c_{n m}}^{T E}=\frac{c \cdot p_{n m}^{\prime}}{2 \pi a \sqrt{\mu_{r} \varepsilon_{r}}} \\
& f_{c_{n m}}^{T M}=\frac{c \cdot p_{n m}}{2 \pi a \sqrt{\mu_{r} \varepsilon_{r}}}
\end{aligned}
$$

where $p_{n m}$ and $p_{n m}^{\prime}$ as tabulated in Table 1 define the $n^{\text {th }}$-zero of the $m^{\text {th }}$-order Bessel function and Bessel function derivative, respectively, $c$ is the light velocity at free space $\left(3 \times 10^{8} \mathrm{~m} / \mathrm{s}\right)$, whilst the integers $m$ and $n$ are related to the number of circumferential variations in the field and the number of radial variations, respectively. From (1) and (2), it should be noted that the dominant mode is the $\mathrm{TE}_{11}$ wave mode, and is normally the one used. Figure 1 shows

Table 1. Value of $p_{n m}^{\prime}$ for TE wave mode and $p_{n m}$ for TM wave mode

\begin{tabular}{|c|c|c|c|c|c|c|}
\hline \multirow{2}{*}{$\boldsymbol{n}$} & \multicolumn{3}{|c|}{ TE wave mode } & \multicolumn{3}{c|}{ TM wave mode } \\
\cline { 2 - 7 } & $\boldsymbol{p}_{\boldsymbol{n} \mathbf{1}}$ & $\boldsymbol{p}_{\boldsymbol{n} \mathbf{2}}$ & $\boldsymbol{p}_{\boldsymbol{n} \mathbf{3}}$ & $\boldsymbol{p}_{\boldsymbol{n} \mathbf{1}}$ & $\boldsymbol{p}_{\boldsymbol{n} \mathbf{2}}$ & $\boldsymbol{p}_{\boldsymbol{n} \mathbf{3}}$ \\
\hline 0 & 3.832 & 7.016 & 10.174 & 2.405 & 5.520 & 8.654 \\
\hline 1 & 1.841 & 5.331 & 8.536 & 3.832 & 7.016 & 10.174 \\
\hline 2 & 3.054 & 6.706 & 9.970 & 5.135 & 8.417 & 11.620 \\
\hline
\end{tabular}


resonance modes consecutively in circular waveguide starting from the $\mathrm{TE}_{11}$ wave dominant mode. Hence, distributions of electric and magnetic field components for the $\mathrm{TE}_{11}$ wave mode, the $\mathrm{TM}_{01}$ wave mode, and the $\mathrm{TE}_{01}$ wave mode in transversal area are illustrated in Figure 2.

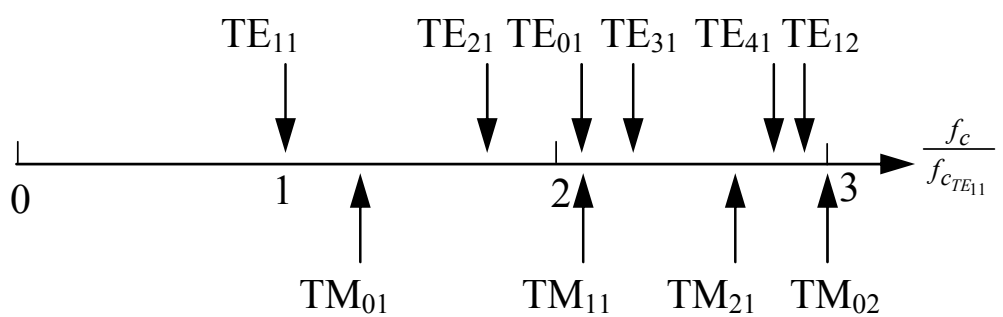

Figure 1. Resonance modes in circular waveguide

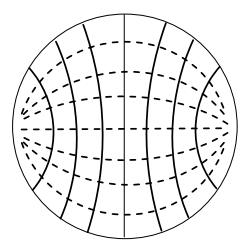

$\mathrm{TE}_{11}$

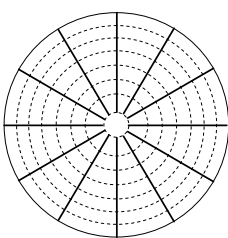

$\mathrm{TM}_{01}$

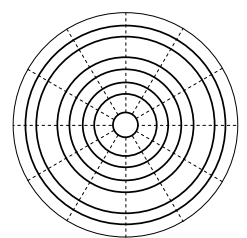

$\mathrm{TE}_{01}$
$\boldsymbol{E}$

H. - -

Figure 2. Field distribution of electric and magnetic field component in transversal area

\section{B. Transmission Loss}

As the signal travels along waveguide, propagating waves suffer from transmission loss. The transmission loss in $\mathrm{THz}$ frequencies is far bigger compared with near-infrared, optical, and radio frequencies. In fact, the transmission loss in $\mathrm{THz}$ frequencies is around the order of $\mathrm{dB} / \mathrm{m}$. One of properties to address this problem is the attenuation constant $(\alpha)$ which commonly arises from metallic surfaces and absorption inside dielectrics. In the hollow metallic circular terahertz waveguide, the attenuation is mostly affected by the metallic surface conductors that construct the inner walls of waveguide. The attenuation constants for the TE and TM wave modes are expressed in (3) and (4), respectively [14].

$$
\begin{aligned}
& \alpha_{m n}^{T E}=\frac{R_{m}}{a Z_{0}}\left(1-\frac{k_{c, m n}^{2}}{k_{0}^{2}}\right)^{-\frac{1}{2}} \cdot\left(\frac{k_{c, m n}^{2}}{k_{0}^{2}}+\frac{n^{2}}{\left(p_{m n}^{\prime}\right)^{2}-n^{2}}\right) \\
& \alpha_{m n}^{T M}=\frac{R_{m}}{a Z_{0}}\left(1-\frac{k_{c, m n}^{2}}{k_{0}^{2}}\right)^{-\frac{1}{2}}
\end{aligned}
$$

where $R_{\mathrm{m}}$ and $Z_{0}$ are the resistive loss and the free space impedance, respectively. Hence $k_{0}$ and $k_{\mathrm{c}}$ are the propagation constant at free space and the $m n$ mode propagation constant, respectively. Since the waves propagate along the waveguide comply the propagation modes, the fields associated with the modes produce currents in the inner walls of waveguide. For imperfect conductor materials $\left(\sigma_{\mathrm{c}}<\infty\right)$, the inner waveguide walls behave like resistors and dissipate energy in the form of heat [15]. This loss is also referred as ohmic loss. Moreover, skin depth factor is also an important matter to keep the propagating wave along the waveguide. The inner walls of waveguide should be in several skin depths such that the distributed wall currents are essentially to be surface currents. This skin depth factor is given in 
(5) [15]. Materials which have better conductivity such as silver, copper, etc. and operate in higher frequency $(\sim \mathrm{THz})$ will have skin depth on the order of nanometer.

$$
\delta=\frac{1}{\sqrt{\pi f \mu \sigma_{c}}}
$$

\section{Numerical Analysis and Discussion}

\section{A. Hollow Metallic Circular Terahertz Waveguide}

The principle to get better transmission is to keep the energy distribution inside the inner area of the waveguide, in other words the transmission energy near the metallic surface of inner waveguide walls has to be minimized. Prior investigating the modification of hollow metallic circular terahertz waveguide using dielectric-lined method, at first, the characteristic of hollow metallic circular terahertz waveguide should be attained to its lowest loss propagation. It is defined based on the mode which is excited inside the waveguide along with the effect of dimensions transition of waveguide diameter. Figure 3 shows the hollow metallic circular terahertz waveguide used for the investigation. The diameter of waveguide $(a)$ is $1.8 \mathrm{~mm}$; therefore the cut-off frequency of dominant mode, i.e. the $\mathrm{TE}_{11}$ wave mode, can be calculated from (1) and is $0.1 \mathrm{THz}$. Hence, the investigation is conducted from in the range of $0.1 \mathrm{THz}$ to $1.0 \mathrm{THz}$. The metallic waveguide wall is chosen using silver with the conductivity $\left(\sigma_{\mathrm{c}}\right)$ of $6.1 \mathrm{x}$ $10^{7} \mathrm{~S} / \mathrm{m}$, consequently the maximum skin depth $(\delta)$ from $(5)$ is about $117 \mathrm{~nm}$ at $0.1 \mathrm{THz}$. The thickness of waveguide walls $\left(t_{w}\right)$ is chosen to be $50 \mu \mathrm{m}$ which is thicker than the calculated skin depth to obtain the better reflectivity response.

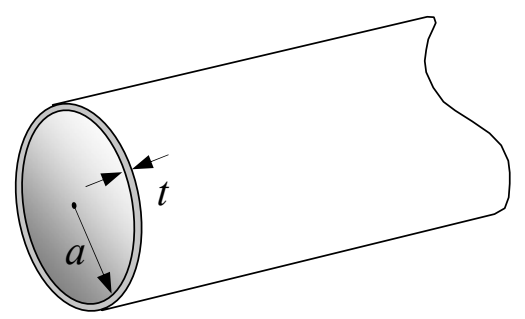

Figure 3. Geometry of hollow metallic circular terahertz waveguide

In the investigation in which the total length of waveguide is $10 \mathrm{~mm}$, the first 2 simple resonance modes, i.e. $\mathrm{TE}_{01}$ and $\mathrm{TM}_{01}$, are observed for analyzing the transmission loss of propagation mode. Both resonance modes are focused for the investigation of transmission loss as they have only radial variation in the fields which are appropriate for the implementation of dielectric-lined method. The analysis is conducted based on forward transmission coefficient $\left(S_{21}\right)$ obtained from $S$-parameters of each propagation mode. The numerical result shows couple of excitations which generate similar waveguide mode with its polarization orthogonal to each other. These modes are also referred as degenerate modes. Both of modes have also similar transmission parameters. Each propagation mode is then identified by matching the vectors pattern of the field components. Figure 4 shows the electric field magnitude and vector in transversal area of circular waveguide for the first 2 simple resonance modes with the $\mathrm{TE}_{11}$ dominant mode as comparison.

From the transmission coefficient $\left(S_{12}\right)$ result depicted in Figure 5, the $\mathrm{TE}_{01}$ wave mode has the transmission loss lower than the $\mathrm{TM}_{01}$ wave mode. This can be understood that the $\mathrm{TE}_{01}$ mode has only tangential electric field component to the conducting wall. Whilst the $\mathrm{TM}_{01}$ wave mode, on other hand it has only normal electric field component affecting higher ohmic loss. The result also complies with the theoretical point of view, i.e. (3) and (4), where the transmission loss or attenuation constant of the $\mathrm{TM}_{01}$ wave mode is higher than of the $\mathrm{TE}_{01}$ wave mode about 7.265 times at $0.4 \mathrm{THz}$ and 9.825 times at $0.6 \mathrm{THz}$. Other modes such as the 
$\mathrm{TE}_{11}$ and $\mathrm{TE}_{21}$ wave modes will have both normal and tangential electric field vector which mostly will contribute even higher ohmic loss. It is also shown that the higher the frequency, the lower the transmission loss of $\mathrm{TE}_{01}$ wave mode. This can be figured out that at the higher frequency the skin depth $(\delta)$ is thinner than at the lower frequency which evokes the penetrating of electric field to the waveguide wall is smaller. As a result, the wave travels along the waveguide suffers smaller transmission loss. The other modes include $\mathrm{TM}_{01}$ wave mode increase their losses as the increase of frequency.
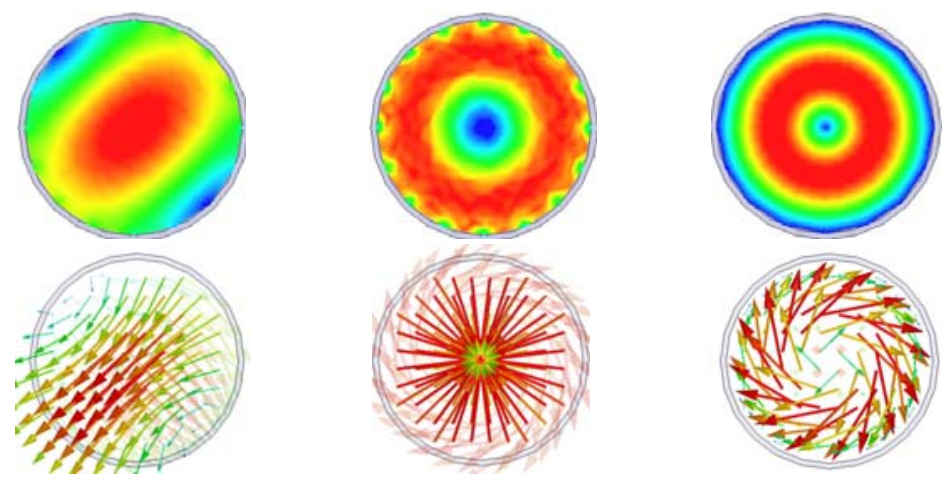

(a) $\mathrm{TE}_{11}$ wave mode (b) $\mathrm{TM}_{01}$ wave mode(c) $\mathrm{TE}_{01}$ wave mode

Figure 4. Distribution of electric field magnitude (up) and vector (bottom) in transversal area

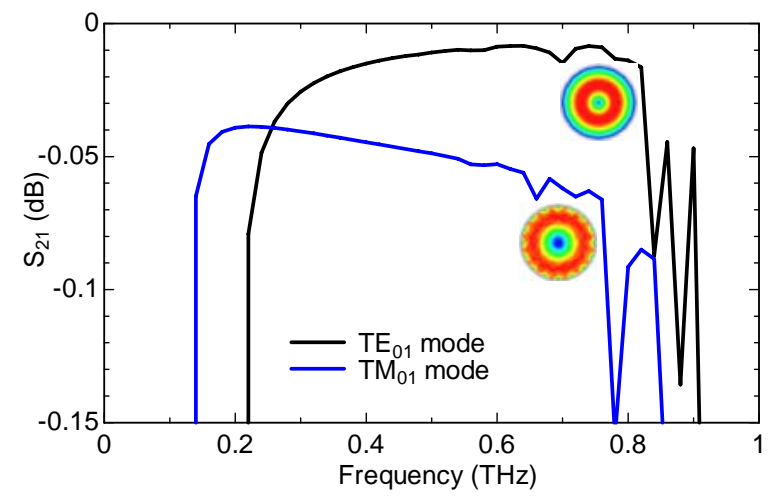

Figure 5. Comparison of transmission loss of $\mathrm{TE}_{01}$ wave mode and $\mathrm{TM}_{01}$ wave mode

From the investigation result, the effect of waveguide diameter is investigated in the $\mathrm{TE}_{01}$ wave mode to obtain its transmission loss characteristic. The diameter of hollow metallic circular terahertz waveguide is varied from $0.5 \mathrm{~mm}$ to $4.0 \mathrm{~mm}$ in which the diameter of $1.8 \mathrm{~mm}$ in previous investigation is used as a reference. The transmission loss of $\mathrm{TE}_{01}$ wave mode becomes lower by the increase of waveguide diameter. In smaller diameters, the transmission loss could be even higher than $3 \mathrm{~dB} / \mathrm{m}$ and have wider bandwidth at higher frequency than the larger ones. The summary of transmission loss for the lowest loss mode is given in Table 1. It notes that the waveguide diameter of $2.0 \mathrm{~mm}$ has better transmission loss characteristic than the other ones with the lowest loss of $0.00475 \mathrm{~dB}(\approx 0.475 \mathrm{~dB} / \mathrm{m})$ at $0.64 \mathrm{THz}$ and the average loss of $1.084 \mathrm{~dB} / \mathrm{m}$. Even though the diameter of $4.0 \mathrm{~mm}$ has the lowest loss as well as the average loss better than the diameter of $2.0 \mathrm{~mm}$, however the larger waveguide diameter has narrower operation bandwidth for the particular modes due to the lower cut-off frequencies of the next higher order mode. 
Table 2. Transmission loss of $\mathrm{TE}_{01}$ wave mode hollow metallic circular terahertz waveguide for different diameters

\begin{tabular}{|c|c|c|c|}
\hline \multirow{2}{*}{$\begin{array}{c}\text { Waveguide } \\
\text { diameter }(\mathrm{mm})\end{array}$} & $\begin{array}{c}\text { Lowest loss } \\
(\mathrm{dB} / \mathrm{m})\end{array}$ & Frequency $(\mathrm{THz})$ & Operation bandwidth \\
\cline { 3 - 4 } & 3.348 & $0.44-0.92$ & 6.376 \\
\hline 1.0 & 0.820 & $0.36-0.84$ & 1.493 \\
\hline 1.6 & 0.833 & $0.32-0.80$ & 1.234 \\
\hline 1.8 & 0.475 & $0.24-0.84$ & 1.084 \\
\hline 2.0 & 0.167 & $0.12-0.40$ & 0.378 \\
\hline 4.0 & \multicolumn{2}{|c}{} \\
\hline
\end{tabular}

\section{B. Dielectric-lined Hollow Metallic Circular Terahertz Waveguide}

The transmission loss in hollow metallic circular terahertz waveguide has been analyzed with the lowest loss operation bandwidth still higher than $1 \mathrm{~dB} / \mathrm{m}$. To minimize the loss, the dielectric material is deposited in the inner surface of metallic waveguide walls, as illustrated in Figure 6. Here, a polymer namely polystyrene (PS) with the refraction index of 1.606 is chosen for the investigation as in practical it can be coated in smooth and uniform distribution. The polystyrene has also low extinction coefficient, so that the dielectric loss can be neglected. The diameter of waveguide $(a)$ is set to be $2.0 \mathrm{~mm}$ as in previous investigation; hence the total length of waveguide is $5 \mathrm{~mm}$ to truncate the need of numerical resources. Similar to the previous investigation, the thickness of metallic waveguide walls $\left(t_{w}\right)$ is $50 \mu \mathrm{m}$; whilst the thickness of polystyrene coating $\left(t_{P S}\right)$ is varied from $5 \mu \mathrm{m}$ to $25 \mu \mathrm{m}$.

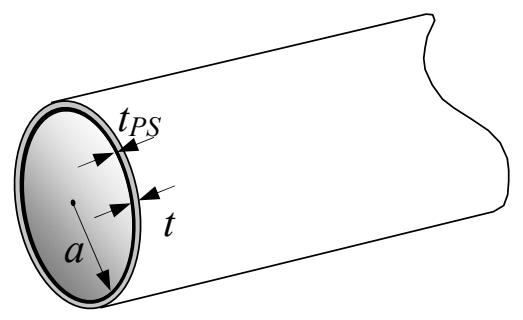

Figure 6. Modified of hollow metallic circular terahertz waveguide by coating polystyrene (PS) in inner surface of waveguide walls

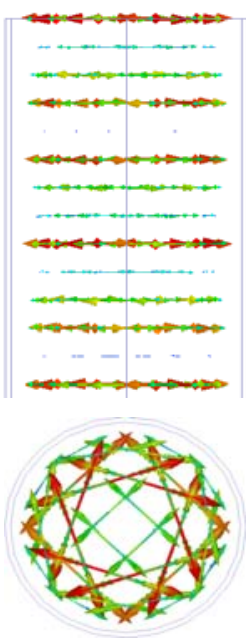

(a) $0 \mu \mathrm{m}$

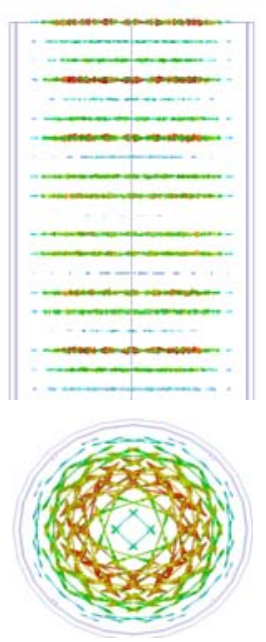

(b) $10 \mu \mathrm{m}$

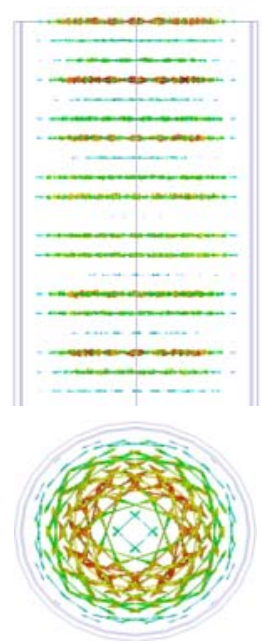

(c) $15 \mu \mathrm{m}$

Figure 7. Distribution of $\mathrm{TE}_{01}$ wave mode electric field vector in longitudinal area (up) and transversal area (bottom) for waveguide with different thickness of polystyrene coating 


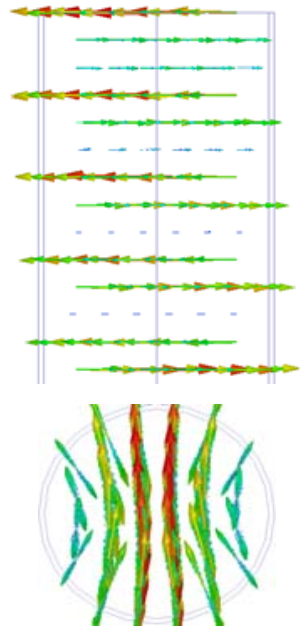

(a) $0 \mu \mathrm{m}$

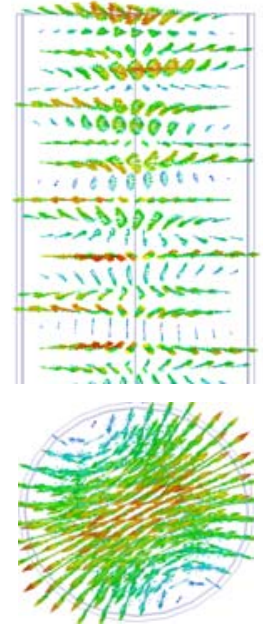

(b) $10 \mu \mathrm{m}$

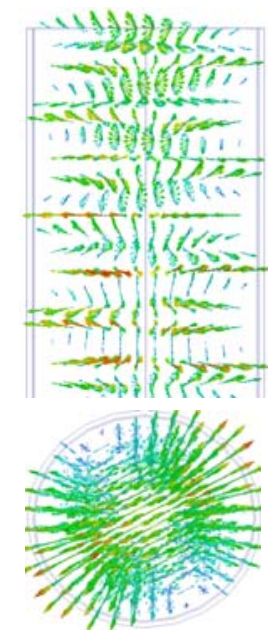

(c) $15 \mu \mathrm{m}$

Figure 8. Distribution of $\mathrm{TE}_{11}$ wave mode electric field vector in longitudinal area (up) and transversal area (bottom) for waveguide with different thickness of polystyrene coating

The numerical result shows that the mode with lowest loss is still the $\mathrm{TE}_{01}$ wave mode. On other hand, the $\mathrm{TM}_{01}$ wave mode increases its loss as the dielectric thickness increases. This can be understood that the polystyrene coating in some thickness can avoids the tangential electric field in penetrating the waveguide wall, therefore, the transmission loss for the $\mathrm{TE}_{01}$ wave mode decreases. It can be shown in Fig, 7 that the amplitude of electric field around the waveguide walls decreases as the existence of polystyrene coating for the $\mathrm{TE}_{01}$ wave mode.

Another important point is the generation of hybrid mode of $\mathrm{TE}_{11}$ dominant mode. From the longitudinal area, the electric field vectors distribution have longitudinal components, while from the transversal area the electric fields are concentrated in the inner area of waveguide especially for thicker polystyrene coating. The mode change is shown in Figure 8. Unfortunately, the mode has still high transmission loss over $3.0 \mathrm{~dB} / \mathrm{m}$ due to the circumferential variation in the electric field near the inner surface of waveguide walls.

Table 3. Transmission loss of $\mathrm{TE}_{01}$ wave mode of metallic circular terahertz waveguide for different thickness of polystyrene coating

\begin{tabular}{|c|c|c|c|c|}
\hline \multirow{2}{*}{$\begin{array}{c}\text { Polystyrene } \\
\text { thickness } \\
(\mu \mathrm{m})\end{array}$} & \multicolumn{2}{|c|}{ Lowest loss } & \multicolumn{2}{|c|}{ Loss of $1 \mathrm{~dB} / \mathrm{m}$} \\
\hline & $\begin{array}{c}\text { Frequency } \\
\text { (THz) }\end{array}$ & $\begin{array}{c}\text { Loss } \\
(\mathrm{dB} / \mathrm{m})\end{array}$ & $\begin{array}{l}\text { Bandwidth } \\
\text { (THz) }\end{array}$ & $\begin{array}{c}\text { Average loss } \\
(\mathrm{dB} / \mathrm{m})\end{array}$ \\
\hline 0 & 0.64 & 0.475 & $0.40-0.68$ & 0.656 \\
\hline 5 & 0.56 & 0.615 & $0.40-0.68$ & 1.485 \\
\hline 10 & 0.72 & 0.459 & $0.40-0.76$ & 0.611 \\
\hline 15 & 0.72 & 0.438 & $0.40-0.76$ & 0.633 \\
\hline 20 & 0.72 & 0.513 & $0.44-0.76$ & 0.756 \\
\hline 25 & 0.60 & 0.562 & $0.44-0.72$ & 0.790 \\
\hline
\end{tabular}

The transmission loss of $\mathrm{TE}_{10}$ wave mode for different thickness of polystyrene coating is summarized in Table 3. It seems that the polystyrene coating of $15 \mu \mathrm{m}$ thick gives better transmission loss of the waveguide below $1 \mathrm{~dB} / \mathrm{m}$ with wider operation bandwidth than the waveguide without coating. However, the average loss of $15 \mu \mathrm{m}$ thickness polystyrene coating is still higher than of $10 \mu \mathrm{m}$ thickness. As the thickness of polystyrene coating increases more than $15 \mu \mathrm{m}$, the average loss also increases due to the additional dielectric loss coming from polystyrene which attenuates the propagated signals through the dielectric material. Furthermore, the thicker polystyrene coating is harder to be realized in order to get smooth and 
uniform distribution. The abnormality result is shown for polystyrene coating thickness of $5 \mu \mathrm{m}$. In this case, the thickness of polystyrene coating is very thin compared with other constructions which probably influences the accuracy of numerical result. Therefore, a high precision of numerical resources is required to solve this construction.

\section{Conclusions}

The transmission loss reduction for hollow metallic circular terahertz waveguide has been investigated by using dielectric-lined method. It has been shown that the increase of waveguide diameter could minimize the transmission loss, however the operation bandwidth became narrower due to the lower cut-off frequencies of the next higher order mode. Whilst, the dielectric-lined method with polystyrene coating has been successfully demonstrated to reduce the transmission loss of circular terahertz waveguide especially for the $\mathrm{TE}_{01}$ wave mode with smaller amplitude of electric field around the inner surface of waveguide walls. It has been shown that the polystyrene coating of $15 \mu \mathrm{m}$ thickness could contribute in reducing the transmission loss of waveguide below $1 \mathrm{~dB} / \mathrm{m}$. In addition, the proposed method was also able manipulating the distribution field pattern of other mode.

\section{Acknowledgements}

This work is partially supported by the Research Grant from Institut Teknologi Bandung (ITB) under the program scheme of Research and Innovation 2013 contract number DIPA023.04.2.189730/2013. The authors wish to thank to Mr. Hardi Nusantara and Dr.-Ing Chairunnisa, both from School of Electrical Engineering and Informatics, Institut Teknologi Bandung (ITB) for useful discussion and strong support.

\section{References}

[1] M.M. Awad and R. A. Cheville, "Transmission terahertz waveguide-based imaging below the diffraction limit," Appl. Phys. Lett., Vol. 86, Issue 22, May 2005.

[2] K.L. Nguyen, M.L. Johns, L.F. Gladden, C.H. Worrall, P. Alexander, S. Barbieri, H.E. Beere, D.A. Ritchie, and E.H. Linfleld, "3D imaging with a terahertz quantum cascade laser," Joint $30^{\text {th }}$ International Conference on Infrared and Millimeter Waves and 13th International Conference on Terahertz Electronics (IRMMW-THz) Proc., Vol. 1, pp. 1112, Virginia, USA, Sep. 2005.

[3] H. Quast, A. Keil, and T. Loffler, "Investigation of foam and glass fiber structures used in aerospace applications by all-electronic 3D Terahertz imaging," $35^{\text {th }}$ International Conference on Infrared Millimeter and Terahertz Waves (IRMMW-THz) Proc., pp. 1-2, Rome, Italy, Sep. 2010.

[4] A. Rahman, "Terahertz spectroscopy brings a new dawn of biological research," Applied Research and Photonics, Inc. (ARP). 2008.

[5] P. Younus, S. Desbarats, E. Bosio, Abraham, J.C. Delagnes, and P. Mounaix, "Terahertz dielectric characterisation of photopolymer resin used for fabrication of $3 \mathrm{D} \mathrm{THz}$ imaging phantoms," Electronics Lett., Vol. 45, Issue 13, pp. 702-703, Jun. 2009.

[6] Y. Otani, T. Sasaki, M. Yuasa,. H. Suga, Kasuga, and H. Ohmori, "THz 3D imaging with phase-shifting interferometry," Conference on Lasers and Electro-Optics Pacific Rim (CLEO-PR) Proc. pp. 1-2, Kyoto, Japan, Jul. 2013.

[7] L. Korneev, A. Bogdanov, Nalivkin, and S. Berezin, "3D imaging system based on FMCW millimeter wave radar," Joint $30^{\text {th }}$ International Conference on Infrared and Millimeter Waves and 13th International Conference on Terahertz Electronics (IRMMWTHz) Proc., Vol. 1, pp. 367-368, Virginia, USA, Sep. 2005.

[8] F. Deicke, W. Fisher, and M. Faulwasser, "Optical wireless communication to ecosystem," Future Network \& Mobile Summit Proc., pp. 1-8, Dresden, Germany, Jul. 2012.

[9] S.P. Jamison, R.W. McGowan and D. Grischkowsky, "Single-mode waveguide propagation and reshaping of sub-ps terahertz pulses in saphire fibers," Appl. Phys. Lett., Vol. 76, Issue. 15, pp. 1987-1989, Apr. 2000. 
[10] T.I. Jeon and D. Grischkowsky, "Direct optoelectronic generation and detection of subps-electrical pulses on sub-mm coaxial transmission lines," Appl. Phys. Lett., Vol. 85, Issue 25, pp. 6092-6094, Dec. 2004.

[11] O. Mitrofanov, R. James, F.A. Fernandez, T.K. Mavrogordatos, and J.A. Harrington, "Reducing transmission losses in hollow THz waveguides," IEEE Trans. on Terahertz Science and Technology. Vol. 1, Issue 1, pp. 124-132, Sep. 2011.

[12] A. Munir and A. Setiawan, "Transmission loss minimization of circular waveguide at terahertz frequencies for imaging application," $8^{\text {th }}$ International Conference on Multimedia Information Technology and Applications (MITA) Proc., pp. 169-172, Beijing, China, Jun. 2012.

[13] H. Nusantara, A. Setiawan, Chairunnisa and A. Munir, "Investigation of dielectric-lined for transmission loss reduction of optical waveguide," $4^{\text {th }}$ International Conference on Electrical Engineering and Informatics (ICEEI) Proc., pp. 1070-1074, Selangor, Malaysia, Jun. 2013.

[14] R.E. Collin, Field theory of guided waves, $2^{\text {nd }}$ Ed., New Jersey: IEEE Press, 1991.

[15] D.M. Pozar, Microwave engineering, $3^{\text {rd }}$ Ed. John Wiley \& Sons, 2005.

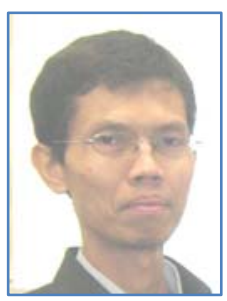

Achmad Munir received the B.E. degree in Electrical Engineering from Institut Teknologi Bandung, Indonesia, in 1995, the M.E. and D.E. degree in science and engineering from Yamaguchi University, Japan, in 2002 and 2005 respectively. From 2005 to 2007, he was a Research Fellow under JSPS fellowship program with department of Electrical and Electronics Engineering, Faculty of Engineering, Yamaguchi University, Japan, working on the artificial materials research, particularly, artificial dielectric and artificial magnetic materials. From 2007 to 2009, he was a Research Fellow with the Institute of Electronics, Communications, and Information Technology, Queens University Belfast, Northern Ireland, United Kingdom, involved in the experimental study of novel nonlinear artificial material including high impedance surface and artificial magnetic conductor for advanced EM applications. In January 2009, he joined the School of Electrical Engineering and Informatics, Institut Teknologi Bandung, Indonesia, as Lecturer. His research interests include linear and nonlinear artificial materials, electromagnetics wave propagation, and microwave devices.

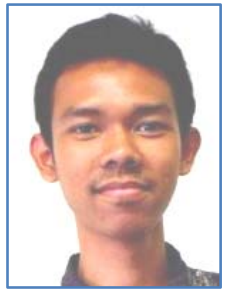

Aryan Setiawan received the B.E. degree in Telecommunication Engineering from School of Electrical Engineering and Informatics, Institut Teknologi Bandung, Indonesia, in 2012. From 2010 to 2011, he was a Young Scientist Exchange Program (YSEP) student with Tokyo Institute of Technology, Japan. His research interests include terahertz and optical waveguide and their numerical analysis. 